\title{
Results of a Comprehensive Infection Control Program for Reducing Surgical-Site Infections in Coronary Artery Bypass Surgery: Further Data From the Authors
}

\author{
Samuel J. McConkey, MB; Paul B. L'Ecuyer, MD; Denise M. Murphy, RN, MPH; \\ Terry L. Leet, PhD; Thoralf M. Sundt, MD; Victoria J. Fraser, MD
}

We welcome Dr. Lee's contribution to the discussion of the issues raised in our article ${ }^{1}$ and value his demonstrated perspicacity and clarity.

The leg wounds of the patients in our study were closed by subcuticular closure with Dexon suture in the majority of cases during the study years. When time constraints dictated, or in particularly obese patients, there was a preference for skin staples. There was no systematic change in surgical techniques with regard to leg-wound closure during the study. It is, however, likely that greater attention was paid to issues such as wound irrigation and wound drainage during the study, as individual surgical assistants responsible for wound closure were receiving feedback regarding their specific surgical-site infection rates.

To detect possible surgical-site infections, we used a prospective cardiothoracic (CT) surgery database and an infection control (IC) database. A coordinator reviewed all cases and all outcomes. The coordinators and IC staff remained the same throughout the study period. IC staff made rounds on the ward and the intensive care unit 2 or 3 times per week. The CT nurses and medical staff called the IC department for every suspicious wound. IC staff reviewed the hospital chart and diagnosis of suspected cases. We monitored antibiotic use, as well as microbiology culture results. The practice of all cardiac surgeons at Barnes Hospital is to see patients 1 month postoperatively, and greater than $90 \%$ are seen here at least once in followup. Infection control nurses at other hospitals call us if they identify an infection thought to be due to an operation in Barnes Hospital.

The primary outcome in this study was total surgicalsite infections. This decreased significantly. We also looked at two subgroups: deep chest infections, as defined in our article, and combined deep and superficial incisional leg infections, referred to in the article as "leg infections." Most of the improvement in infection rates occurred in leg infections (Table 1 ). We did not show a statistically significant decrease in deep chest infections; however, our sample size is too small for the study to have the power to detect a clinically important change in this outcome. Superficial incisional chest infection rates increased for the first 3 years and decreased to a rate above the initial rate in the last year of the study.

Our term deep chest infections is not standard terminology, but it is unambiguously defined in the "Methods" section of the article in terms of the Centers for Disease Control and Prevention categories outlined by Dr. Lee. It includes mediastinal and sternal infections, but excludes cutaneous and subcutaneous infections of the sternal incision.

We have one reservation about the validity of the analysis and have addressed this as described below. In general, any regression model assumes that the outcome of interest for a particular individual is independent of all other individuals in the study sample. Based on this assumption, one calculates the errors of the estimates, and thus one generates a probability value for hypothesis testing. However, in a situation like the one in our study, the independence of the events cannot be taken for granted. Is it possible that when one individual gets a surgical-site infection others are at higher risk? This certainly would be biologically plausible. Events that occur over a period of time often have a tendency toward related outcomes for events that are in close temporal proximity. This is timeseries auto-correlation. If it were present in a series of cases such as we present, it would invalidate the hypothesis testing in the logistic regression model. We have tested for the presence of auto-correlation in the residual values for the models described in the article. In this case, none was found. However, it is possible that the model could be sensitive to small degrees of auto-correlation that were not statistically significant.

From the Division of Infectious Diseases (Drs. McConkey, L'Ecuyer, and Fraser), the Division of Cardiothoracic Surgery (Dr. Sundt), Washington University School of Medicine; the Department of Infection Control (Ms. Murphy), Barnes Hospital; Saint Louis University School of Public Health (Dr. Leet), St Louis, Missouri.

Address reprint requests to Victoria J. Fraser, MD, Division of Infectious Diseases, Washington University School of Medicine, 660 S Euclid Ave, Campus Box 8051, St Louis, MO 63110.

99-ED-171. McConkey SJ, L'Ecuyer PB, Murphy DM, Leet TL, Sundt TM, Fraser VJ. Results of a comprehensive infection control program for reducing surgical-site infections in coronary artery bypass surgery: further data from the authors. Infect Control Hosp Epidemiol 2000;21:791-792. 
TABLE 1

Number of Patients With Surgical-Site Infections of Different Types After Coronary Artery Bypass Graft (CABG) Surgery

\begin{tabular}{|c|c|c|c|c|c|c|c|c|}
\hline \multirow[b]{2}{*}{ Location of Infection } & 1991 & \multicolumn{2}{|c|}{1992} & \multicolumn{2}{|c|}{1993} & \multicolumn{2}{|c|}{1994} & Totals \\
\hline & $N(\%)$ & $\mathbf{N}$ & $(\%)$ & & $(\%)$ & & $(\%)$ & $N(\%)$ \\
\hline Superficial incisional chest & $17(3.98)$ & 37 & $(6.12)$ & 36 & $(6.25)$ & 29 & $(4.65)$ & $119(5.33)$ \\
\hline Deep chest & $11(2.58)$ & 12 & (1.98) & 9 & $(1.56)$ & 10 & (1.61) & $42(1.88)$ \\
\hline Superficial incisional leg & $25(5.85)$ & 51 & (8.43) & 34 & (5.9) & 17 & (2.73) & $127(5.69)$ \\
\hline Deep incisional leg & $4(0.94)$ & 16 & $(2.64)$ & 9 & (1.56) & 0 & $(0)$ & $29(1.3)$ \\
\hline Total leg infection & $29(6.79)$ & 67 & (11.1) & 43 & $(7.47)$ & 17 & (2.73) & $156(6.99)$ \\
\hline Total infection (chest and leg) & $53(12.4)$ & 106 & (17.5) & 81 & $(14.1)^{\star}$ & 51 & (8.19) & $291(13.0)$ \\
\hline Number of CABG surgeries & 427 & 605 & & 576 & & 623 & & 2,231 \\
\hline
\end{tabular}

Total infection is the total number of patients with a surgical-site infection. Some patients had infection at both chest and leg, so the total infection does not always equal the sum of the first four rows. * The rate of total infection for 1993 was $14.1 \%$, not $16.1 \%$ as is stated in Table 3 of the article. ${ }^{1}$

We agree with Dr. Lee that we should have been more consistent in terminology. We agree that factors other than the infection control program could have caused the infection rates to decrease. In the study, we tried to control for severity of illness and other factors to facilitate meaningful comparison of infection rates. In a study such as this, which uses individuals from a different time-period as controls, it is possible that some other factor changed over the time of the study and caused the observed change in rates.

Our goal is to improve the quality of care that patients receive. This requires measuring important out- comes, so that they can be compared in a meaningful way, and evaluating interventions that may improve the outcomes. Both of these are difficult tasks. Within the limitations of what is possible, we believe that our study provides evidence that infection control interventions have a beneficial effect for patients who have coronary artery bypass graft surgery.

\section{REFERENCE}

1. McConkey SJ, L'Ecuyer PB, Murphy DM, Leet TL, Sundt TM, Fraser VJ. Results of a comprehensive infection control program for reducing surgical-site infections in coronary artery bypass surgery. Infect Control Hosp Epidemiol 1999;20:533-538. 